\title{
Current indications for transplantation: stratification of severe heart failure and shared decision-making
}

\author{
Darko Vucicevic ${ }^{1}$, Lily Honoris ${ }^{1}$, Federica Raia ${ }^{1,2}$, Mario Deng $^{1}$ \\ ${ }^{1}$ David Geffen School of Medicine at UCLA, Ronald Reagan UCLA Medical Center, Los Angeles, CA, USA; ${ }^{2}$ Graduate School of Education \& \\ Information Studies (GSEIS), UCLA, Los Angeles, CA, USA \\ Correspondence to: Mario Deng, MD, FACC, FESC. David Geffen School of Medicine at UCLA, Ronald Reagan UCLA Medical Center, 100 UCLA \\ Medical Plaza, Suite 630 East, Los Angeles, CA 90095, USA. Email: mdeng@mednet.ucla.edu.
}

\begin{abstract}
Heart failure (HF) is a complex clinical syndrome that results from structural or functional cardiovascular disorders causing a mismatch between demand and supply of oxygenated blood and consecutive failure of the body's organs. For those patients with stage D HF, advanced therapies, such as mechanical circulatory support (MCS) or heart transplantation (HTx), are potentially life-saving options. The role of risk stratification of patients with stage D HF in a value-based healthcare framework is to predict which subset might benefit from advanced HF (AdHF) therapies, to improve outcomes related to the individual patient including mortality, morbidity and patient experience as well as to optimize health care delivery system outcomes such as costeffectiveness. Risk stratification and subsequent outcome prediction as well as therapeutic recommendationmaking need to be based on the comparative survival benefit rationale. A robust model needs to (I) have the power to discriminate (i.e., to correctly risk stratify patients); (II) calibrate (i.e., to show agreement between the predicted and observed risk); (III) to be applicable to the general population; and (IV) provide good external validation. The Seattle Heart Failure Model (SHFM) and the Heart Failure Survival Score (HFSS) are two of the most widely utilized scores. However, outcomes for patients with HF are highly variable which make clinical predictions challenging. Despite our clinical expertise and current prediction tools, the best short- and long-term survival for the individual patient, particularly the sickest patient, is not easy to identify because among the most severely ill, elderly and frail patients, most preoperative prediction tools have the tendency to be imprecise in estimating risk. They should be used as a guide in a clinical encounter grounded in a culture of shared decision-making, with the expert healthcare professional team as consultants and the patient as an empowered decision-maker in a trustful safe therapeutic relationship.
\end{abstract}

Keywords: Heart failure (HF); stage D; risk stratification; heart transplantation (HTx); mechanical circulatory support (MCS); palliation therapy; shared decision making

Submitted Aug 08, 2017. Accepted for publication Oct 16, 2017.

doi: $10.21037 /$ acs.2017.12.01

View this article at: http://dx.doi.org/10.21037/acs.2017.12.01

\section{Introduction}

Heart failure (HF) is a complex clinical syndrome that results from structural or functional cardiovascular disorders causing a mismatch between demand and supply of oxygenated blood and consecutive failure of the body's organs. Despite significant advances in understanding HF pathophysiology and development of new treatment modalities, HF is still associated with high morbidity and mortality, causing a significant burden in the healthcare system.

In technologically advanced modern medicine, advanced HF (AdHF) therapies, such as mechanical circulatory support (MCS) and heart transplantation (HTx), are treatment options considered for patients at the most advanced stages of the disease (1), characterized as stage D or AdHF (2). These therapies have the potential to improve survival and enhance quality of life (3). It is paramount to appropriately select patients that would benefit the most 
from AdHF therapies in order to (I) optimize the benefit for the individual patient and (II) responsibly utilize a scarce resource of society such as HTx because, despite increasing number of patients with HF, the number of HTx performed per year in United States remains relatively constant.

The goal of this review is to identify current indications for HTx, discuss risk stratification in patients with stage D $\mathrm{HF}$, examine patient selection criteria for advanced therapies and critically review the process of shared decision-making in identifying the best recommendation consistent with the patient's preferences, based on the practice of one of the world's premier AdHF/MCS/HTx-programs.

\section{Epidemiology of AdHF}

Approximately 6.5 million people in the United States are affected by HF (4). The lifetime risk of developing HF is at least 1 in 5 for men and women above 45 years of age (5). It is estimated that the prevalence of patients living with $\mathrm{HF}$ will increase by $46 \%$ by the year 2030 , achieving epidemic proportions (6). Furthermore, HF imposes a great burden to society with an estimated annual cost of $\$ 30.7$ billion in 2012 with projected annual cost of almost $\$ 70$ billion by the year 2030 (4). Although survival of patients with HF have improved with time due to advances in therapy, the death rate remains unacceptably high at approximately $50 \%$ within 5 years from the time of initial diagnosis (4). In the US, there is an estimated 300,000 individuals annually who suffer from AdHF or stage D HF which designates patients with truly refractory HF (2). These patients usually require inotropic support to maintain hemodynamics and have very poor prognosis. In a recent clinical trial, $20 \%$ of patients with Stage D HF requiring inotropic support randomized to medical therapy were alive at 1-year post-randomization (7). For those patients with stage D HF, advanced therapies, such as MCS or HTx, are potentially life-saving options.

\section{Pathophysiology \& neuro-endocrino-immunology of AdHF}

$\mathrm{HF}$ is initiated by various myocardial injury mechanisms and decompensated HF leads to reduced blood pressure (BP), cardiac output $(\mathrm{CO})$ and oxygen $\left(\mathrm{O}_{2}\right)$ delivery. Changes in metabolism result in overall catabolic/anabolic imbalance, leading to cardiac cachexia, frailty and muscle wasting. Additionally, patients with stage D HF tend to develop iron deficiency anemia, resulting in further impairment of $\mathrm{O}_{2}-$ delivery to the tissues (8). Through chronic upregulation of sympathetic nervous system (SNS) and reninangiotensin-aldosterone system (RAAS) activity, patients with HF try to maintain a compensated state, yet further myocardial injury leads to $\mathrm{HF}$ progression, reduced $\mathrm{O}_{2}$ delivery to organs/tissues and organ dysfunction (OD) (9). This triggers immune system activation that provides shortterm compensation for the failing heart in an attempt to reverse OD. However, this immune system activation also coincides with progressive OD in the kidneys, liver, bone marrow and brain, profound metabolic derangements, creating a milieu similar to systemic diseases (10-16).

Elderly and frail patients with stage D HF who fail optimal medical management (OMM) often suffer from malnutrition, immune dysfunction, and poor infection coping potential. They are therefore at increased risk for OD and death after MCS or HTx surgery. HF-related preoperative immunologic impairment is a component of poor outcomes after MCS and HTx, owing to the known associations between increased age, $\mathrm{T}$ cell and innate immune cell dysfunction (17-19), increased numbers of terminally differentiated $\mathrm{T}$ cells (20), immune-senescence (deficient replicative ability), and immune-exhaustion (impaired antigen response) $(21,22)$.

\section{Therapeutic options \& outcomes in AdHF}

\section{Medical management}

During the last five decades, significant advances have been made in medical management of patients with systolic $\mathrm{HF}$ with the introduction of beta blockers, angiotensin converting enzyme inhibitors, angiotensin receptor blockers, aldosterone antagonists and most recently neprilysin inhibitors. These medications were shown in clinical trials to improve the survival and reduce morbidity in this patient population (23-25). However, patients with stage D HF often cannot tolerate OMM by guideline directed medical therapy (GDMT) and do not derive the same benefit as patients with less advanced disease.

\section{High risk coronary artery bypass surgery (CABG)}

In individuals with low ejection fraction and ischemic disease, high risk CABG can be considered. This procedure requires high level of surgical expertise and should be performed only in high volume centers. Furthermore, due to the complexity of these patients and an increased risk of clinical decompensation, advanced therapies should be 
discussed with the patients a priori and their candidacy for MCS and HTx should also be assessed. High risk CABG can modestly improve cardiac function and functional status in selected patients (26). The impact on survival remains unclear, with some studies showing benefit and others showing no difference in mortality (27). Selection of patients that would benefit the most from high risk revascularization remains a topic of debate in the HF community.

\section{Anti-arrhythmia device therapy}

Patients with stage D HF are at an increased risk of sudden cardiac death (SCD) from ventricular tachyarrhythmia, thus anti-arrhythmia device therapy is an integral part of their management. Introduction of internal cardioverter defibrillator (ICD) for primary prevention of SCD was proven to be of great benefit with reduction in mortality by $31 \%$ in 20 months in patients with history of $\mathrm{MI}$ and $\mathrm{EF}<30 \%$ (28). Furthermore, in patients with $\mathrm{EF}<35 \%$, regardless of etiology and mild to moderate symptoms, ICD implantation decreases mortality by $23 \%$ over 5 years (29). ACC/AHA HF guidelines recommends ICD implantation in all patients with ejection fraction of $<30 \%$ and New York Heart Association (NYHA) class I symptoms and in those with EF $<35 \%$ with NYHA class II and III symptoms (23). This therapy is, however, reserved for patients with a projected survival of more than 1 year which precludes some of the patients with very advanced disease from receiving an ICD. Cardiac dyssynchrony is also found to be an important factor that impacts efficiency of cardiac function. Cardiac resynchronization therapy (CRT) has been used in patients with wide QRS complex and left bundle branch block (LBBB) pattern on EKG. The benefits are seen in improvement of ventricular contractility and $\mathrm{EF}$, reduction in secondary mitral regurgitation, reversal of remodeling and at times, improvement in BP. Despite reducing mortality and improving quality of life in selected patients, around 30\% of individuals receiving this therapy derive no benefit or experience worsening of their symptoms (30). Similar to ICD, patients with stage D HF are often considered to be too sick to benefit from CRT and therefore their treatment is limited to advanced therapies (MCS and HTx) or palliative care (23).

\section{Current indications for HTx}

Since its first introduction in 1967, HTx offers an unparalleled survival benefit in select patients with stage D
HF, and remains the gold standard of treatment.

Stage D HF is defined as refractory HF and often accompanied by the following parameters: repeated $(>2)$ hospitalizations or ED visits for HF in the past year, worsening renal function, unintentional weight loss $>10 \%$ (cardiac cachexia), intolerance to medical therapy due to hypotension and/or worsening renal function, persistent dyspnea/fatigue, hyponatremia and escalating use of diuretics $(>160 \mathrm{mg} / \mathrm{d}$ and/or use of supplemental metolazone therapy) and frequent ICD shocks $(23,31)$.

ACC/AHA guidelines designate a class I indication for HTx only in carefully selected patients with stage D HF despite GDMT, device, and surgical management. Current graft survival rates with advances in immunosuppressive therapy are $85-90 \%, 75-80 \%$, and $70-75 \%$ in adults at $1-, 3-$, and 5-year respectively, and a median survival of 11-13 years (23). Additionally, HTx offers significant improvement in quality of life and patient satisfaction. However, although potentially curative, HTx requires long term immunosuppressive therapy, putting patients at risk of infection. Cardiac allograft vasculopathy, a poorly understood immunologic process, is the most feared complication of HTx and is the most common cause of graft failure. Annually, there are approximately 3,000 HTx performed in the U.S. and the number of donors have remained steady for decades. Needless to say, the main limitation of HTx is the lack of available donors.

\section{Current indications for MCS}

As shown in destination MCS trials, mortality in patients with stage D HF receiving medical therapy is unacceptably high and other therapeutic options need to be considered for these patients (7).

Long-term MCS was introduced in early 1980s as an option for patients with stage D HF. These devices can be implanted as bridge to transplantation (BTT) or destination therapy (DT) and can provide $\mathrm{LV}$ or biventricular support. Several devices are approved by Food and Drug Administration (FDA) for use in stage D HF patients that fulfill strict selection criteria. MCS therapies have been shown in clinical trials to improve survival, with survival rate in current era of $80 \%$ and $70 \%$ at 1- and 2-year respectively. Survival rate of MCS as DT therapy is $75-80 \%$ and $55-60 \%$ at $1-$ and 3 -year respectively. With advances in technology, MCS devices are getting smaller, easier to implant and offer significant improvement in quality of life. However, despite numerous benefits these therapies 
provide, rates of complication including bleeding, driveline infection, thrombosis and stroke remain high (32).

\section{Clinical risk prediction \& comparative survival benefit rationale}

The role of risk stratification of patients with stage D HF in a value-based healthcare framework is to predict which subset might benefit from AdHF therapies, to improve outcomes related to the individual patient including mortality, morbidity and patient experience as well as to optimize health care delivery system outcomes such as costeffectiveness. Risk stratification and subsequent outcome prediction, as well as therapeutic recommendation-making needs to be based on the comparative survival benefit rationale (33). Determining which patients with stage D HF would derive the most benefit from advanced therapies and recognizing the appropriate timing for administering these therapies before development of profound multi$\mathrm{OD}$, remains one of the most important aspects of care in this patient population. Prognosis can be improved with early diagnosis, optimization of medical therapy, MCS and HTx. However, outcomes for patients with HF are highly variable, which makes clinical predictions challenging. Despite our clinical expertise and current prediction tools, the therapy with the best short- and long-term survival for the individual patient, particularly the sickest patient, is not easy to identify $(18,32,34-46)$. A key reason is that among the most severely ill patients, most preoperative prediction tools in HF, critical care medicine and cardiac surgery have the tendency to be imprecise in estimating risk, particularly underestimating risk $(41,47)$. Several parameters including clinical characteristics, hemodynamic markers, serum biomarkers, and therapeutic treatment are used in HF models as clinical risk predictors to prognosticate HF outcomes. Some commonly used parameters and models will be discussed below.

B-type natriuretic peptide (BNP) is a widely used biomarker for diagnosis and prognosis of HF (34). Troughton hypothesized that titration of treatment to reduce plasma N-BNP concentrations is beneficial in patients with systolic HF. However, the use of BNP as prognostic indicator in AdHF population has not been rigorously validated. Peak $\mathrm{VO}_{2}$ has been found to be a prognostic predictor of survival and is often used as an initial screen to identify patients with AdHF (48). Low Peak $\mathrm{O}_{2}$ consumption is an indicator of poor heart function and $\mathrm{VO}_{2} \max \leq 14 \mathrm{~mL} / \mathrm{kg} / \mathrm{min}$ is a criteria for heart transplant consideration (49). However, peak $\mathrm{VO}_{2}$ may be influenced by several confounding factors such as age, gender, motivation, anemia, body weight and muscle deconditioning.

Some clinical variables have been identified to be associated with increased mortality in patients with HF and several HF models and risk assessment scores have been developed, all in the hope of predicting HF survival. Risk models, or a statistically derived framework from robust datasets, can be useful in predicting outcomes. A robust model needs to (I) have the power to discriminate (i.e., to correctly risk stratify patients); (II) calibrate (i.e., to show agreement between the predicted and observed risk); (III) be applicable to the general population; and (IV) provide good external validation (50). The UCLA HF risk model provides a simple prognostic information in both men and women with AdHF using four common clinical variables: BNP, peak $\mathrm{VO}_{2}$, NYHA class, and angiotensinconverting enzyme inhibitor/angiotensin receptor blocker (44). However, its suitability as a selection tool for MCS and HTx is inconsistent and high-risk discrimination was poor in an external validation cohort (40). Seattle Heart Failure Model (SHFM) and the Heart Failure Survival Score (HFSS) are two of the most widely utilized scores. Both models have their own intrinsic limitations but both have been shown to give fairly reasonable estimates of 1-year survival and have been recommended as consideration for HTx selection (49).

\section{The SHFM}

The SHFM provides risk strata, an estimation of 1-, 2and 5-year survival rates, a mean life expectancy and an estimated survival curve, using 20 commonly obtained clinical, pharmacologic, device and laboratory parameters.

The web-based calculator (http://www. SeattleHeartFailureModel.org) provides an automated risk percentage. The SHFM, described by Levy et al. in 2006 , is widely referenced and has been validated in numerous settings $(38,51,52)$. However, its derivation and validation were carried out in datasets obtained from clinical trials which did not include the use of beta-blockers and defibrillators/CRT which are the standard of care for HF patients in the modern era. Its cohort was mostly Caucasian with a wide range of NYHA classes from I-IV and left ventricular ejection fractions (LVEF) with $1 / 3$ of patients in one validation cohort had an LVEF of $>40 \%$. Later studies also found differential effects of some HF 


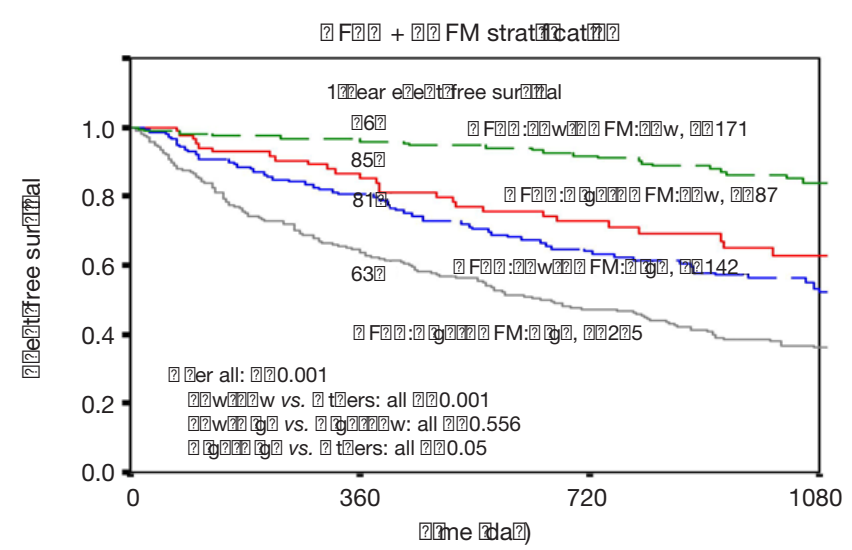

Figure 1 Kaplan-Meier event-free survival curves stratified by combined HFSS + SHFM groups [adapted from reference (55)]. Since heart transplantation confers a 1-year survival of $85-90 \%$, patients with a Heart Failure Survival Score (HFSS) high risk and/ or Seattle Heart Failure Model (SHFM) high risk may derive a survival benefit from heart transplantation.

medications between Caucasian and African-American patients which were not addressed in the SHFM $(53,54)$. Furthermore, $98 \%$ of events in the SHFM derivation and validation databases were death, rather than HTx or MCS implantation. Kalogeropoulos (51) assessed the performance of the SHFM in patients with AdHF referred for transplant evaluation and found that overall the SHFM provided good discrimination between low-risk versus highrisk patients (Figure 1). However, the model overestimated survival and underestimated absolute risk among high-risk black population and patients with implanted devices (51). These limitations in the original SHFM cohorts question the applicability of the model in the sicker and more heterogeneous AdHF population.

\section{HFSS}

The HFSS model includes seven parameters: resting heart rate (HR); mean BP (mBP); LVEF; serum sodium; presence or absence of ischemic heart disease; presence or absence of intraventricular conduction defect (IVCD); and peak $\mathrm{VO}_{2}$. This score stratifies its cohort into three distinct strata: low risk (HFSS $\geq 8.10$ ), medium risk (HFSS 7.20 to 8.09 ), and high risk (HFSS $\leq 7.19$ ). Event-free survival rates at 1 year for the low-, medium-, and high-risk HFSS strata were $93 \% \pm 2 \%, 72 \% \pm 5 \%$, and $43 \% \pm 7 \%$, respectively (Figure 1). Patients in the medium or high-risk strata were advised to consider cardiac transplantation listing and those in the low risk category are monitored on OMM unless their clinical condition deteriorates. HFSS performs better than the peak $\mathrm{VO}_{2}$ alone (56), and has been revalidated in the era of Beta blocker therapy (57), in women (58) and in African American and Hispanic patients (59). In today's device era, HFSS still outperforms the peak $\mathrm{VO}_{2}$ in risk stratification for AdHF patients in the presence of an ICD and/or CRT. It has been proposed that peak $\mathrm{VO}_{2}<10 \mathrm{~mL} / \mathrm{kg} / \mathrm{min}$ rather than the traditional cutoff value $<14 \mathrm{~mL} / \mathrm{min} / \mathrm{kg}$ may be more useful for risk stratification in the device era (55). The main criticism of HFSS lies in its requirement of peak $\mathrm{VO}_{2}$ measurement, which has many confounders and requires specialized equipment and expert interpretation. However, peak $\mathrm{VO}_{2}$ assessment is usually available in tertiary centers where most AdHF therapies are also offered. We hypothesize that the inability to exercise or inability to reach the anaerobic threshold (a respiratory exchange ratio $>1$ ) may indicate that the patient might be too sick or too frail to undergo AdHF therapies.

\section{Comparing SFHM and HFSS}

SHFM and HFSS are widely used risk stratification models. They have both been broadly validated and have excellent prognostic value. SHFM uses 20 convenient clinical parameters which are readily available. Although cumbersome, it may be appropriate as an initial patient selection screening tool. HFSS is more practical since it utilizes only seven parameters but they include peak $\mathrm{VO}_{2}$ measurement. A head to head comparison of HFSS and SHFM on seven hundred fifteen consecutive patients with systolic HF referred for heart transplant evaluation shows modest correlation $(\mathrm{R}=-0.48, \mathrm{P}<0.001)$ (Figure 1). However, this correlation factor is misleading, given the different variables and clinical settings from which these two models were derived. There is, however, concordance in the 1-year event-free survival of $89 \%, 72 \%$ and $60 \%$, respectively, for the low-, medium- and high-risk HFSS strata, and 93\%, $76 \%$, and $58 \%$, respectively, for the low-, medium- and high-risk SHFM strata. This reflects the degree of accuracy in prognostication of each model. When the models were combined, the area under the curve for combined outcome of death/LVAD/transplantation was 0.77 and 0.76 , respectively for 1- and 2-year, which is markedly better than either model alone. If a patient falls into a combined lowrisk HFSS and low-risk SHFM category, the 1-year eventfree survival is $96 \%$ which is higher than that in low-risk HFSS (89\%) and low-risk SHFM (93\%) group separately. 
Therefore, low-risk scores on both risk models provide further reassurance to defer AdHF therapy (Figure 1). On the other hand, the addition of one score to the other may be especially helpful in risk stratifying patients in the medium risk category where at times clinical decision making falls into the grey zone (Figure 1) (55). The validity of these two models prompted their inclusion in the HTx guidelines, which recommend cardiopulmonary exercise test in addition to either (SHFM) score of $<80 \%$ or a HFSS in the high/medium risk range be used as cut points for $\mathrm{HTx}$ listing (class IIb, level of evidence: C) (49).

\section{MCS risk prediction}

MCS, such as durable LVAD, is an alternative AdHF therapy, which can be used as a BTT or as DT. If seen as a part of a continuum of AdHF therapy, the question arises if LVAD therapy needs its own separate risk prediction model. The answer largely lies on the intrinsic nature of the device and the complications encountered with LVAD, namely the tight balance between bleeding risk and clotting risk. Nonetheless, several MCS predictive models have been proposed. It is important to reflect that the MCS model at this time is not used to accept or deny patients of AdHF therapies. It is still generally used as a preoperative risk assessment tool.

Previously, one of the often-used risk scores was the Destination Therapy Risk Score (DTRS), which was developed as part of a U.S. FDA mandated postmarket surveillance of the pulsatile flow HeartMate XVE. However, the DTRS was recently shown to provide poor discrimination for BTT patients and only modest discrimination for DT patients supported with continuous flow LVADs (60). The Interagency Registry for Mechanically Assisted Circulatory Support (INTERMACS) classification system, although not designed as a predictive model, has been proposed to classify patient risk according to pre-operative clinical acuity with reasonable prognostic discrimination (61). The HeartMate Risk Score (HMRS) was derived from clinical trial patients enrolled in the HMII in BTT and DT clinical trials and it is intended to predict risk in the current era of continuous flow LVAD. It provides a simple clinical prediction rule based on patients' age, international normalized ratio, albumin, creatinine, and the implant center's volume. However, this score excluded patients who required acute MCS, other than an intraaortic balloon pump (45). HMRS has been validated using the INTERMACS database. Among 10,847 patients, the
HMRS exhibits moderate discrimination in both, short term 90-day and long-term 2-year mortality. Relative risk of 90-day mortality is 2.8 times greater with patients in the highest HMRS category compared to those in the lowest HMRS category (13.0\% vs. 4.7\%; $\mathrm{P}<0.001)(62)$.

\section{Outcome prediction research}

Most clinicians use the "eyeball test" to determine which patients they believe are frail. "Frailty" is a term often referred to patients with stage D HF since they may be elderly and/or suffer from cardiac cachexia. This is because there are no widely accepted frailty parameters in the HF community. We know from patients who are undergoing cardiac surgery that frailty is associated with adverse outcomes. Flint proposed in 2012 that there might be a subset of frail patients who are responsive to MCS and will continue to thrive whereas another group may not (18). Frailty is a reflection of biological age and not chronological age. It is often thought of as a constellation of subclinical physiological insults in multiple organ systems which makes patients more vulnerable in times of physiologic stress. A study done on 99 patients undergoing DT-LVAD using 31 deficits based on ability to perform activities of daily living (ADLs) and other clinical factors showed that there was a $>3$-fold increased risk of death in patients in the highest frailty tertile compared with those who were not frail (lowest tertile) (19).

The NIH-United States Critical Illness and Injury Trials (USCIIT) Group invited the Deng lab in 2008 to develop a peripheral blood mononuclear cell (PBMC)-gene expression profiling (GEP) biomarker test to better understand HFrelated frailty and OD, diagnose and predict outcomes, and treat HF-related OD. This genomic test, which is under development at UCLA, was named MyLeukoMAP ${ }^{\mathrm{TM}}$. It is expected to predict more precisely the comparative survival benefit of $\mathrm{HTx} / \mathrm{MCS}$ over OMM than current clinical tests alone (63-65).

As a long-term vision, this new blood test will assist, as part of a multidimensional prediction model, the HFspecialist in recommending the best treatment option, either optimal medical management, HTx, MCS or palliation care to an individual patient for the best outcome.

\section{Shared decision making in stage D HF- relational medicine paradigm}

Healthcare providers have an obligation to provide 
information on the natural course of end stage HF, diagnostic tests, risk and benefits of various treatment options, costs and anticipated outcomes. This information should be offered in a way that is easily comprehensible to the patients and their families and in a culturally sensitive manner. Shared decision making is grounded in the principle of a person's autonomy and has been described as "an approach where clinicians and patients share the best available evidence when faced with the task of making decisions, and where patients are supported to consider options, to achieve informed preferences" (66). The clinical decisionmaking challenge at the time of evaluation in our stage D $\mathrm{HF}$ practice often comes as a big shock to patients. The choice often culminates between all that high-tech modern medicine has to offer and compassionate end of life care, both of which are equally daunting considerations. This ultimate scenario is medically, ethically and economically challenging. This life-changing discussion deserves the best evidence-based decision-making support that personalized precision medicine research has to offer to enable a practice of modern medicine that lives up to the highest humanistic expectations that society entrusts us with (67).

Shared decision making, with the expert healthcare professional team consulting about the different options and the empowered patient making decisions in a trustful safe therapeutic relationship, is key for a humanistically sound concept of the practice of modern medicine. This concept which we have coined Relational Medicine $^{\mathrm{TM}}$ (68) is grounded in the basic human right of personhood for all stakeholders, patients, caregivers and healthcare professionals alike.

\section{Case illustration}

CG is a 50-year-old male with a history of non-ischemic cardiomyopathy with an EF of 20\%, NYHA class III/ACC stage $\mathrm{C} \mathrm{HF}$ and left ventricular end diastolic diameter of $9.4 \mathrm{~cm}$. He has a prior history of cardiac arrest and ICD placement for secondary prevention of SCD. He has a progressive decline in his functional status, and undergoes cardiopulmonary stress test as part of his AdHF evaluation. According to SHFM, his 1-year survival is $94.4 \%$. However, he develops ventricular tachyarrhythmia during his cardiopulmonary stress test so HFSS cannot be calculated. $\mathrm{He}$ has a right heart catheterization which showed normal wedge pressure and a cardiac index of 2.5. Although these parameters lend adequate reassurance of a high 1-year survival rate, there should be a healthy skepticism in such a rosy computerized outlook in a patient with a $9.4 \mathrm{~cm}$ dilated left ventricle, history of ventricular tachyarrhythmia and a history of cardiac arrest whom clinicians intuitively consider as high-risk patient.

\section{Discussion}

The role of risk stratification of patients with stage $\mathrm{D} H \mathrm{HF}$ is to predict which patients might benefit from AdHF therapies. However, clinical outcomes for patients diagnosed with HF are highly variable which makes formulating risk stratification models challenging. Two patients with the same ejection fraction can have a different functional status, and different clinical outcomes. Accurate risk stratification of patients with stage D HF remains difficult as illustrated in our case presentation. Various risk stratifications models using clinical data have been proposed to better identify patients who would most benefit from advanced therapies. Multiple other factors such as absence of social support, psychological suitability and frailty are associated with poor outcomes after HTx and MCS but they are not incorporated in any clinical prediction score. All of these factors are needed to approach AdHF therapy candidacy as "whole person" instead of a set of clinical data.

Timing of AdHF therapies before development of profound multi-organ dysfunction remains one of the most important aspects of care in the AdHF patient population. A patient with a low ejection fraction can remain in NYHA class II/III for years and may not be "sick enough" to warrant AdHF evaluation and there is no straightforward clinical parameter that predicts if and when the patient will progress to end stage HF. At the other end of the spectrum, many patients have a "crashing and burning" (INTERMACS I) presentation as their initial HF diagnosis which puts them at the highest risk for adverse outcomes. Healthcare providers are obliged to respect the sanctity of human lives and it is our goal to improve the quality of life of our patients. As such, it is often difficult to recommend palliative care to patients at the highest risk for adverse outcomes.

Patients evaluated for AdHF therapies are selected after careful consideration from a multidisciplinary team in which psychosocial factors are considered in addition to clinical data. Selection of patients who would most benefit from HTx and MCS remains challenging and requires experience and ethical and social considerations. 


\section{Conclusions}

Risk stratification and clinical decision making involving the AdHF or stage D HF patient population is complex and multifaceted since the clinical course for patients with HF is highly variable. Although cardiac transplantation remains the gold standard for patients with stage D HF, the urgent need and lack of available donors make MCS an attractive and viable option, both as a BTT and DT. Various risk stratifications models have been proposed to predict preand postoperative mortality, each with inherent limitations. They should be used as a guide and not a replacement for sound clinical judgment in a clinical encounter that is grounded in a culture of shared decision-making, with the expert healthcare professional team members as consultants about the different options and the patient as empowered decision-maker in a trustful safe space of the therapeutic relationship.

\section{Acknowledgements}

Funding: The study obtained funding by UCLA NIH R21 1R21HL120040-01 (Principal Investigator M Deng), UCLA R01 (Joint Principal Investigator M Deng), UCLA R01 (Co-Investigator M Deng), UCLA DOM Internal Funds and the Advanced HF Research Gift to Columbia University (Philip Geier, John Tocco and Robert Milo) and Advanced HF Research Gift to UCLA (Juan Mulder, Peter Schultz, Larry Layne).

\section{Footnote}

Conflicts of Interest: The authors have no conflicts of interest to declare.

\section{References}

1. Deng MC, Naka Y. Mechanical circulatory support therapy in advanced heart failure. London: Imperial College Press, 2007.

2. Hunt SA, Abraham WT, Chin MH, et al. 2009 Focused update incorporated into the ACC/AHA 2005 Guidelines for the Diagnosis and Management of Heart Failure in Adults A Report of the American College of Cardiology Foundation/American Heart Association Task Force on Practice Guidelines Developed in Collaboration With the International Society for Heart and Lung Transplantation. J Am Coll Cardiol 2009;53:e1-90.
3. Lund LH, Edwards LB, Dipchand AI, et al. The Registry of the International Society for Heart and Lung Transplantation: Thirty-third Adult Heart Transplantation Report-2016; Focus Theme: Primary Diagnostic Indications for Transplant. J Heart Lung Transplant 2016;35:1158-69.

4. Benjamin EJ, Blaha MJ, Chiuve SE, et al. Heart Disease and Stroke Statistics-2017 Update: A Report From the American Heart Association. Circulation 2017;135:e146-603.

5. Huffman MD, Berry JD, Ning H, et al. Lifetime risk for heart failure among white and black Americans: cardiovascular lifetime risk pooling project. J Am Coll Cardiol 2013;61:1510-7.

6. Heidenreich PA, Albert NM, Allen LA, et al. Forecasting the impact of heart failure in the United States: a policy statement from the American Heart Association. Circ Heart Fail 2013;6:606-19.

7. Stevenson LW, Miller LW, Desvigne-Nickens P, et al. Left ventricular assist device as destination for patients undergoing intravenous inotropic therapy: a subset analysis from REMATCH (Randomized Evaluation of Mechanical Assistance in Treatment of Chronic Heart Failure). Circulation 2004;110:975-81.

8. Doehner W, Frenneaux M, Anker SD. Metabolic impairment in heart failure: the myocardial and systemic perspective. J Am Coll Cardiol 2014;64:1388-400.

9. Mann DL, Zipes DP, Libby P, et al. Braunwald's heart disease: a textbook of cardiovascular medicine. Tenth edition. Philadelphia: Elsevier Saunders, 2015.

10. Levine B, Kalman J, Mayer L, et al. Elevated Circulating Levels of Tumor-Necrosis-Factor in Severe Chronic Heart-Failure. N Engl J Med 1990;323:236-41.

11. Deng MC, Erren M, Lutgen A, et al. Interleukin-6 correlates with hemodynamic impairment during dobutamine administration in chronic heart failure. Int J Cardiol 1996;57:129-34.

12. Caruso R, Trunfio S, Milazzo F, et al. Early expression of pro- and anti-inflammatory cytokines in left ventricular assist device recipients with multiple organ failure syndrome. ASAIO J 2010;56:313-8.

13. Caruso R, Verde A, Cabiati M, et al. Association of preoperative interleukin-6 levels with Interagency Registry for Mechanically Assisted Circulatory Support profiles and intensive care unit stay in left ventricular assist device patients. J Heart Lung Transplant 2012;31:625-33.

14. Kaur K, Dhingra S, Slezak J, et al. Biology of TNFalpha and IL-10, and their imbalance in heart failure. Heart Fail Rev 2009;14:113-23. 
15. Soejima H, Irie A, Fukunaga T, et al. Osteopontin expression of circulating $\mathrm{T}$ cells and plasma osteopontin levels are increased in relation to severity of heart failure. Circ J 2007;71:1879-84.

16. Mann DL. Inflammatory mediators and the failing heart: past, present, and the foreseeable future. Circ Res 2002;91:988-98.

17. Dorshkind K, Montecino-Rodriguez E, Signer RA. The ageing immune system: is it ever too old to become young again? Nat Rev Immunol 2009;9:57-62.

18. Flint KM, Matlock DD, Lindenfeld J, et al. Frailty and the selection of patients for destination therapy left ventricular assist device. Circ Heart Fail 2012;5:286-93.

19. Dunlay SM, Park SJ, Joyce LD, et al. Frailty and outcomes after implantation of left ventricular assist device as destination therapy. J Heart Lung Transplant 2014;33:359-65.

20. Larbi A, Fulop T. From "truly naive" to "exhausted senescent" T cells: when markers predict functionality. Cytometry A 2014;85:25-35.

21. Wherry EJ. T cell exhaustion. Nat Immunol 2011;12:492-9.

22. Burton DG, Faragher RG. Cellular senescence: from growth arrest to immunogenic conversion. Age (Dordr) 2015;37:27.

23. Yancy CW, Jessup M, Bozkurt B, et al. 2013 ACCF/AHA guideline for the management of heart failure: a report of the American College of Cardiology Foundation/American Heart Association Task Force on Practice Guidelines. J Am Coll Cardiol 2013;62:e147-239.

24. Yancy CW, Jessup M, Bozkurt B, et al. 2017 ACC/ AHA/HFSA Focused Update of the 2013 ACCF/AHA Guideline for the Management of Heart Failure: A Report of the American College of Cardiology/American Heart Association Task Force on Clinical Practice Guidelines and the Heart Failure Society of America. J Am Coll Cardiol 2017;70:776-803.

25. Adigopula S, Vivo RP, DePasquale EC, et al. Management of ACCF/AHA Stage C heart failure. Cardiol Clin 2014;32:73-93, viii.

26. Elefteriades JA, Tolis G Jr, Levi E, et al. Coronary artery bypass grafting in severe left ventricular dysfunction: excellent survival with improved ejection fraction and functional state. J Am Coll Cardiol 1993;22:1411-7.

27. Chareonthaitawee P, Gersh BJ, Araoz PA, et al. Revascularization in severe left ventricular dysfunction: the role of viability testing. J Am Coll Cardiol 2005;46:567-74.

28. Moss AJ, Hall WJ, Cannom DS, et al. Improved survival with an implanted defibrillator in patients with coronary disease at high risk for ventricular arrhythmia. Multicenter Automatic Defibrillator Implantation Trial Investigators. N Engl J Med 1996;335:1933-40.

29. Bardy GH, Lee KL, Mark DB, et al. Amiodarone or an implantable cardioverter-defibrillator for congestive heart failure. N Engl J Med 2005;352:225-37.

30. Auricchio A, Prinzen FW. Non-responders to cardiac resynchronization therapy: the magnitude of the problem and the issues. Circ J 2011;75:521-7.

31. Russell SD, Miller LW, Pagani FD. Advanced heart failure: a call to action. Congest Heart Fail 2008;14:316-21.

32. Kirklin JK, Naftel DC, Pagani FD, et al. Seventh INTERMACS annual report: 15,000 patients and counting. J Heart Lung Transplant 2015;34:1495-504.

33. Deng MC, De Meester JM, Smits JM, et al. Effect of receiving a heart transplant: analysis of a national cohort entered on to a waiting list, stratified by heart failure severity. Comparative Outcome and Clinical Profiles in Transplantation (COCPIT) Study Group. BMJ 2000;321:540-5.

34. Troughton RW, Frampton CM, Yandle TG, et al. Treatment of heart failure guided by plasma aminoterminal brain natriuretic peptide (N-BNP) concentrations. Lancet 2000;355:1126-30.

35. Gardner RS, Ozalp F, Murday AJ, et al. N-terminal pro-brain natriuretic peptide. A new gold standard in predicting mortality in patients with advanced heart failure. Eur Heart J 2003;24:1735-43.

36. Doust JA, Pietrzak E, Dobson A, et al. How well does B-type natriuretic peptide predict death and cardiac events in patients with heart failure: systematic review. BMJ 2005;330:625.

37. Aaronson KD, Schwartz JS, Chen TM, et al. Development and prospective validation of a clinical index to predict survival in ambulatory patients referred for cardiac transplant evaluation. Circulation 1997;95:2660-7.

38. Levy WC, Mozaffarian D, Linker DT, et al. The Seattle Heart Failure Model: prediction of survival in heart failure. Circulation 2006;113:1424-33.

39. Ketchum ES, Moorman AJ, Fishbein DP, et al. Predictive value of the Seattle Heart Failure Model in patients undergoing left ventricular assist device placement. J Heart Lung Transplant 2010;29:1021-5.

40. Sartipy U, Goda A, Mancini DM, et al. Assessment of a University of California, Los Angeles 4-variable risk score for advanced heart failure. J Am Heart Assoc 2014;3:e000998. 
41. Sartipy U, Goda A, Yuzefpolskaya M, et al. Utility of the Seattle Heart Failure Model in patients with cardiac resynchronization therapy and implantable cardioverter defibrillator referred for heart transplantation. Am Heart J 2014;168:325-31.

42. Martínez-Sellés M, Vidan MT, Lopez-Palop R, et al. End-stage heart disease in the elderly. Rev Esp Cardiol 2009;62:409-21.

43. Smits JM, de Vries E, De Pauw M, et al. Is it time for a cardiac allocation score? First results from the Eurotransplant pilot study on a survival benefit-based heart allocation. J Heart Lung Transplant 2013;32:873-80.

44. Chyu J, Fonarow GC, Tseng CH, et al. Four-variable risk model in men and women with heart failure. Circ Heart Fail 2014;7:88-95.

45. Cowger J, Sundareswaran K, Rogers JG, et al. Predicting survival in patients receiving continuous flow left ventricular assist devices: the HeartMate II risk score. J Am Coll Cardiol 2013;61:313-21.

46. Kormos RL, Teuteberg JJ, Pagani FD, et al. Right ventricular failure in patients with the HeartMate II continuous-flow left ventricular assist device: incidence, risk factors, and effect on outcomes. J Thorac Cardiovasc Surg 2010;139:1316-24.

47. Ad N, Holmes SD, Patel J, et al. Comparison of EuroSCORE II, Original EuroSCORE, and The Society of Thoracic Surgeons Risk Score in Cardiac Surgery Patients. Ann Thorac Surg 2016;102:573-9.

48. Mancini DM, Eisen H, Kussmaul W, et al. Value of peak exercise oxygen consumption for optimal timing of cardiac transplantation in ambulatory patients with heart failure. Circulation 1991;83:778-86.

49. Mehra MR, Canter CE, Hannan MM, et al. The 2016 International Society for Heart Lung Transplantation listing criteria for heart transplantation: A 10-year update. J Heart Lung Transplant 2016;35:1-23.

50. Moons KG, Kengne AP, Woodward M, et al. Risk prediction models: I. Development, internal validation, and assessing the incremental value of a new (bio)marker. Heart 2012;98:683-90.

51. Kalogeropoulos AP, Georgiopoulou VV, Giamouzis G, et al. Utility of the Seattle Heart Failure Model in patients with advanced heart failure. J Am Coll Cardiol 2009;53:334-42.

52. May HT, Horne BD, Levy WC, et al. Validation of the Seattle Heart Failure Model in a community-based heart failure population and enhancement by adding B-type natriuretic peptide. Am J Cardiol 2007;100:697-700.
53. Taylor JS, Ellis GR. Racial differences in responses to drug treatment: implications for pharmacotherapy of heart failure. Am J Cardiovasc Drugs 2002;2:389-99.

54. Taylor AL, Cohn JN, Worcel M, et al. The AfricanAmerican Heart Failure Trial: background, rationale and significance. J Natl Med Assoc 2002;94:762-9.

55. Goda A, Lund LH, Mancini D. The Heart Failure Survival Score outperforms the peak oxygen consumption for heart transplantation selection in the era of device therapy. J Heart Lung Transplant 2011;30:315-25.

56. Lund LH, Aaronson KD, Mancini DM. Validation of peak exercise oxygen consumption and the Heart Failure Survival Score for serial risk stratification in advanced heart failure. Am J Cardiol 2005;95:734-41.

57. Koelling TM, Joseph S, Aaronson KD. Heart failure survival score continues to predict clinical outcomes in patients with heart failure receiving beta-blockers. J Heart Lung Transplant 2004;23:1414-22.

58. Green P, Lund LH, Mancini D. Comparison of peak exercise oxygen consumption and the Heart Failure Survival Score for predicting prognosis in women versus men. Am J Cardiol 2007;99:399-403.

59. Goda A, Lund LH, Mancini DM. Comparison across races of peak oxygen consumption and heart failure survival score for selection for cardiac transplantation. Am J Cardiol 2010;105:1439-44.

60. Teuteberg JJ, Ewald GA, Adamson RM, et al. Risk assessment for continuous flow left ventricular assist devices: does the destination therapy risk score work? An analysis of over 1,000 patients. J Am Coll Cardiol 2012;60:44-51.

61. Boyle AJ, Ascheim DD, Russo MJ, et al. Clinical outcomes for continuous-flow left ventricular assist device patients stratified by pre-operative INTERMACS classification. J Heart Lung Transplant 2011;30:402-7.

62. Adamo L, Tang Y, Nassif ME, et al. The HeartMate Risk Score Identifies Patients With Similar Mortality Risk Across All INTERMACS Profiles in a Large Multicenter Analysis. JACC Heart Fail 2016;4:950-8.

63. Sinha A, Shahzad K, Latif F, et al. Peripheral blood mononuclear cell transcriptome profiles suggest T-cell immunosuppression after uncomplicated mechanical circulatory support device surgery. Hum Immunol 2010;71:164-9.

64. Bondar G, Cadeiras M, Wisniewski N, et al. Comparison of whole blood and peripheral blood mononuclear cell gene expression for evaluation of the perioperative inflammatory response in patients with advanced heart 
failure. PLoS One 2014;9:e115097.

65. Wisniewski N, Bondar G, Rau C, et al. Integrative model of leukocyte genomics and organ dysfunction in heart failure patients requiring mechanical circulatory support: a prospective observational study. BMC Med Genomics 2017;10:52.

66. Elwyn G, Frosch D, Thomson R, et al. Shared decision making: a model for clinical practice. J Gen Intern Med
2012;27:1361-7.

67. Raia F, Deng MC. Artificial heart pumps: bridging the gap between science, technology and personalized medicine by relational medicine. Future Cardiol 2017;13:23-32.

68. Raia F, Deng M. Relational Medicine: Personalizing Modern Healthcare - The Practice of High-Tech Medicine as a RelationalAct. 1st Edition. WSPC/Imperial College Press, 2014.

Cite this article as: Vucicevic D, Honoris L, Raia F, Deng M. Current indications for transplantation: stratification of severe heart failure and shared decision-making. Ann Cardiothorac Surg 2018;7(1):56-66. doi: 10.21037/acs.2017.12.01 